\title{
Uso de pausas activas en la modalidad de enseñanza virtual para promover el bienestar emocional en jóvenes universitarios durante el periodo de pandemia
}

\section{USE OF ACTIVE BREAKS IN THE VIRTUAL TEACHING MODALITY TO PROMOTE EMOTIONAL WELL-BEING IN YOUNG UNIVERSITY STUDENTS DURING THE PANDEMIC PERIOD}

Carolina Lovón Cueva

Universidad de Lima

cmlovon@ulima.edu.pe

\section{RESUMEN}

Durante el periodo de confinamiento en el Perú del 2020 por el COVID-19, siete de diez personas han tenido alguna afección mental (Siete de cada 10 peruanos han visto afectada su salud mental durante pandemia, 2020). Muchos peruanos desarrollaron casos de estrés, ansiedad o tensiones emocionales, 0 bien aumentó el nivel de estas enfermedades. Entre estas personas, se encuentran también los estudiantes universitarios, quienes se enfrentaron a un sistema de educación virtual que priorizó la transmisión de contenidos y conocimientos sin considerar la sobrecarga académica que repercute en la salud emocional de los jóvenes. Ante esta nueva experiencia, el presente estudio busca analizar la aplicación de pausas activas en la enseñanza virtual de la Universidad de Lima (UL) como estrategia pedagógica que propicia espacios de bienestar emocional para regular los estados de ánimo, evitar la ansiedad, la angustia, el estrés, la frustración o la tristeza. La investigación recoge testimonios de los estudiantes del Programa de Estudios Generales de la UL durante sus clases virtuales del periodo 2020-2. Los resultados muestran que mejoraron sus competencias emocionales y aumentó su bienestar personal.

PALABRAS CLAVE: bienestar emocional, pausas activas, estrategia pedagógica
ABSTRACT

During the period of confinement in Peru in 2020 by COVID-19, seven out of ten people have had some mental condition. Many Peruvians developed cases of stress, anxiety, or emotional tensions; or in other cases, the level of these illnesses increased. Among these people, there are also university students, who faced a virtual education system, which prioritized the transmission of content and knowledge without considering the academic overload that affects young people's emotional health. This study seeks to analyze the application of active breaks in virtual teaching at the University of Lima (UL), as a pedagogical strategy that favors spaces of emotional well-being, regulates moods, and avoids anxiety, and anguish, stress, frustration or sadness. The research collects testimonies from the UL General Studies Program students during their virtual classes in the 2020-2 period. The results show that students improved their emotional competencies and increased their personal well-being.

KEYWORDS: emotional well-being, active breaks, pedagogical strategies 


\section{INTRODUCCIÓN}

El 15 de marzo del 2020 el presidente del Perú y el Consejo de Ministros decretaron el estado de emergencia por la pandemia del COVID-19. Durante este periodo, se dictaminó el aislamiento social obligatorio para evitar la expansión del coronavirus. En un primer momento, esto implicó la paralización total de ciertos sectores, tales como el económico, social, cultural y educacional, entre otros. Luego, en diferentes fases, se reactivaron sus funciones, y se aplicaron planes de contingencia y mejora continua ante la coyuntura social. En contexto, la educación universitaria también tuvo que adaptarse a la nueva coyuntura e incorporó una modalidad de enseñanza-aprendizaje virtual.

Cuando las diversas universidades del país emplearon la modalidad de dictado virtual, tuvieron que recurrir a sus recursos pedagógicos más próximos. Así, se evidencia un dictado virtual tradicional o con un enfoque de contenidos que no contempla estrategias pedagógicas que atiendan no solo al desarrollo de estos, sino al proceso emocional del estudiante frente al nuevo sistema educativo. La ausencia de una integración de estrategias pedagógicas en un ambiente de dictado virtual es una falencia del sistema educativo que nos lleva a cuestionar el beneficio académico y el bienestar emocional de los estudiantes, dimensión que ha cobrado creciente relevancia durante la pandemia.

Pocos autores han concentrado su estudio en la preocupación de que si las estrategias pedagógicas virtuales están contemplando la salud mental y emocional de los participantes en el periodo de pandemia. Arana (2020) señala que tanto los profesores como los estudiantes han tenido una sobrecarga académica y que han experimentado más inestabilidad emocional durante las clases virtuales. Al respecto, indica que esto puede deberse a la mediación pedagógica, es decir que los facilitadores no estarían contemplando dinámicas de clase que favorezcan la relación emocional del estudiantado, lo que desencadena cargas de estrés y frustración entre los participantes. Alvarado (2021) también reconoce la importancia de la aplicación de la educación emocional durante la pandemia. Él sostiene que implementar estrategias didácticas con competencias emocionales durante el proceso de enseñanza-aprendizaje virtual favorece la estabilidad emocional dentro del marco de la situación sanitaria. Estas prácticas permitirán regular las emociones negativas como el estrés, enojo, tristeza, frustración, miedo y ansiedad. Alvarado (2021) concluye que se podrá favorecer el desarrollo personal y profesional al emplear estas prácticas de estrategias didácticas con competencias emocionales. Es necesario mencionar que, en el contexto educativo de América del Sur, el Ministerio de Educación de Chile elaboró recursos para la contención emocional en el periodo de crisis sanitaria. Entre estos recursos, se entregaron los siguientes:

Una guía con orientaciones para el autocuidado y el bienestar socioemocional en el contexto de crisis sanitaria, asociado a una Bitácora Docente para el desarrollo de un trabajo personal y autónomo de aprendizaje socioemocional a partir 
de su propia experiencia, para que luego puedan transmitirlo a sus estudiantes. (Comisión Económica para América Latina y el Caribe; Organización de las Naciones Unidas para la Educación, la Ciencia y la Cultura, 2020)

Considerando un enfoque cuantitativo, esta investigación estudia la importancia de aplicar pausas activas como estrategias pedagógicas en la enseñanza-aprendizaje de los estudiantes universitarios de la Universidad de Lima, las cuales fueron empleadas en clases virtuales en el contexto de la cuarentena por el COVID-19. En términos metodológicos, se aplicó una encuesta para identificar la importancia del uso de las pausas activas en el dictado virtual para mejorar el bienestar emocional en jóvenes universitarios durante el periodo de pandemia.

Investigaciones como la presente son cruciales para comprender las prácticas pedagógicas virtuales en función del bienestar personal y emocional de los estudiantes en el periodo de cuarentena. Además, podría ser fuente de investigaciones futuras para proponer, innovar o mejorar las diferentes estrategias pedagógicas en un contexto de enseñanza virtual, como también se puede continuar un estudio sobre el mejoramiento del rendimiento académico a partir de estas pausas activas (véase la figura 10).

El presente estudio está estructurado de la siguiente manera: marco conceptual, marco metodológico, discusión y conclusiones.

\section{PAUSAS ACTIVAS Y BIENESTAR EMOCIONAL EN LA EDUCACIÓN VIRTUAL ACTUAL}

Andina, Agencia Peruana de Noticias, publicó que siete de diez personas han tenido alguna afección mental durante el periodo de confinamiento en el Perú por el COVID-19 (Siete de cada 10 peruanos han visto afectada su salud mental durante pandemia, 2020). Muchos peruanos desarrollaron casos de estrés, ansiedad o tensiones emocionales, o bien aumentó el nivel de estas enfermedades. Entre estas personas, se encuentran también los estudiantes universitarios (Lovón y Cisneros, 2020), quienes se enfrentaron a un sistema de educación virtual que priorizó la transmisión de contenidos sin considerar la sobrecarga académica que repercute en la salud emocional de los jóvenes. A partir de la coyuntura, se reconoce como prioritario atender el bienestar personal y emocional de los estudiantes universitarios a partir de estrategias pedagógicas como las pausas activas. La aplicación de estas permitirá regular los estados de ánimo, evitar la ansiedad, la angustia, el estrés, la frustración o la tristeza; de esta manera, los estudiantes mejorarán sus competencias emocionales y aumentarán su bienestar personal.

En un inicio, las pausas activas fueron empleadas en el ámbito laboral como breves descansos para realizar actividades físicas con la finalidad de prevenir el estrés y trastornos musculoesqueléticos, mejorar el desempeño y la creatividad, o reducir la fatiga. En el campo educativo también se han incorporado las pausas activas como estrategias 
pedagógicas para mejorar el rendimiento académico, favorecer la actividad física y generar un estilo de vida afectivo y saludable. Dichos objetivos mencionados son contemplados en la mayoría de estudios pedagógicos sobre las pausas activas (Florines, 2017; Lorca, 2016; Jiménez y Monroy, 2015), pero no han desarrollado con amplitud la relación de esta estrategia con el bienestar emocional. Otros estudios han abordado la importancia de la aplicación de estas dinámicas para promover el bienestar emocional, pero no las han implementado en un contexto pedagógico. Por ejemplo, Meza (2010) describe la relación del bienestar psicológico con actividades o ejercicios propios de la danza. Con la Escala de Bienestar Psicológico (EBP) y una encuesta, se determinó que existe mayor bienestar psicológico en personas que emplean en su vida diaria ciertas prácticas de danza. También Rodríguez (2016), en su laboratorio-taller, identifica los aportes efectivos entre la relación de dinámicas de movimiento con las competencias intrapersonales e interpersonales de la inteligencia emocional. El estudio concluye que la práctica de movimientos favorece el desarrollo y estado emocional.

En el marco de la emergencia sanitaria, se acentuó la preocupación por el bienestar emocional frente a una educación virtual. En este sentido, los estudios de Arana (2020) y Alvarado (2021), mencionados anteriormente, son los que se aproximan a validar la importancia de estrategias o dinámicas que promuevan el bienestar emocional en un ambiente de enseñanza-aprendizaje virtual durante el periodo de pandemia.

\section{LA UNIVERSIDAD DE LIMA Y LA EDUCACIÓN VIRTUAL ACTUAL}

Desde sus inicios, la Universidad de Lima (UL) ha orientado la formación de estudiantes en principios democráticos, y ha buscado una preparación de profesionales capacitados en constante actualización para contribuir al progreso y desarrollo de la sociedad peruana. Asimismo, la UL ha concentrado su interés por los recursos educativos en ciencia y tecnología, y el haber integrado programas y servicios para el bienestar de la comunidad universitaria.

Frente a la crisis sanitaria, la UL tuvo que pasar el dictado de clases presenciales a una modalidad virtual. Además, ante esta coyuntura, tampoco se desatendieron los programas y servicios de bienestar, que también tuvieron su apertura en transmisión virtualizada. Si bien antes de la pandemia la UL ya brindaba capacitaciones a sus docentes sobre el uso de herramientas digitales educativas, estas fueron constantes durante la crisis sanitaria.

En este contexto, es preciso articular el uso de estrategias pedagógicas que favorezcan al bienestar emocional del estudiante durante las clases virtuales. 


\section{MÉTODO}

Este estudio se realizó con cuarenta estudiantes del ciclo 2020-2 de la Universidad de Lima, quienes ya habían experimentado clases virtuales desde el ciclo anterior. Como instrumento principal de esta investigación, se empleó una encuesta y su aplicación fue individual. Los ítems fueron divididos en dos escalas: la primera parte atendió al bienestar personal durante las clases virtuales en el periodo de pandemia, mientras que la segunda parte abordó el conocimiento de las pausas activas y la percepción después de emplearlas en las clases virtuales.

Se aplicaron tres sesiones de pausas activas en la primera unidad del curso y tres en la segunda unidad. En algunas sesiones se emplearon las pausas activas vinculadas al contenido de la clase y, en otras sesiones, se usaron como activación para dar inicio a la clase o como recuperación de energía después de la clase.

\section{RESULTADOS}

¿Crees que durante el periodo de pandemia tuviste sobrecarga académica?

40 respuestas

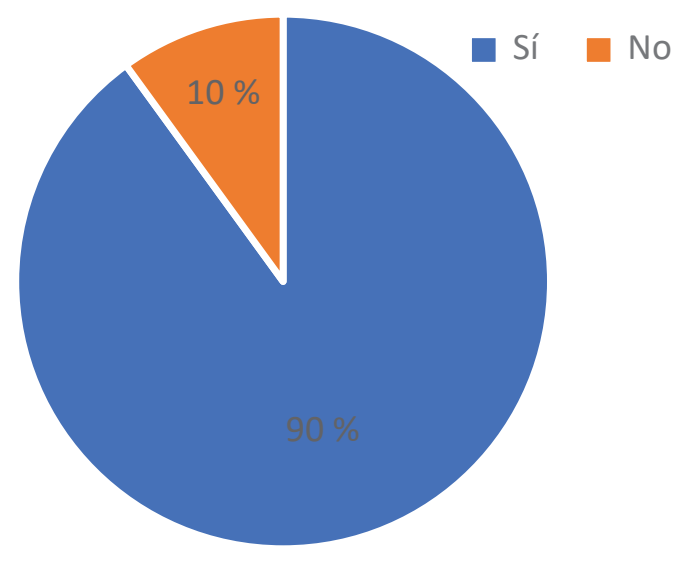

Figura 1. Cantidad de estudiantes que afirman haber tenido sobrecarga académica en pandemia

Elaboración propia 
A partir de la siguiente escala, ¿cómo te sientes durante tus clases virtuales?

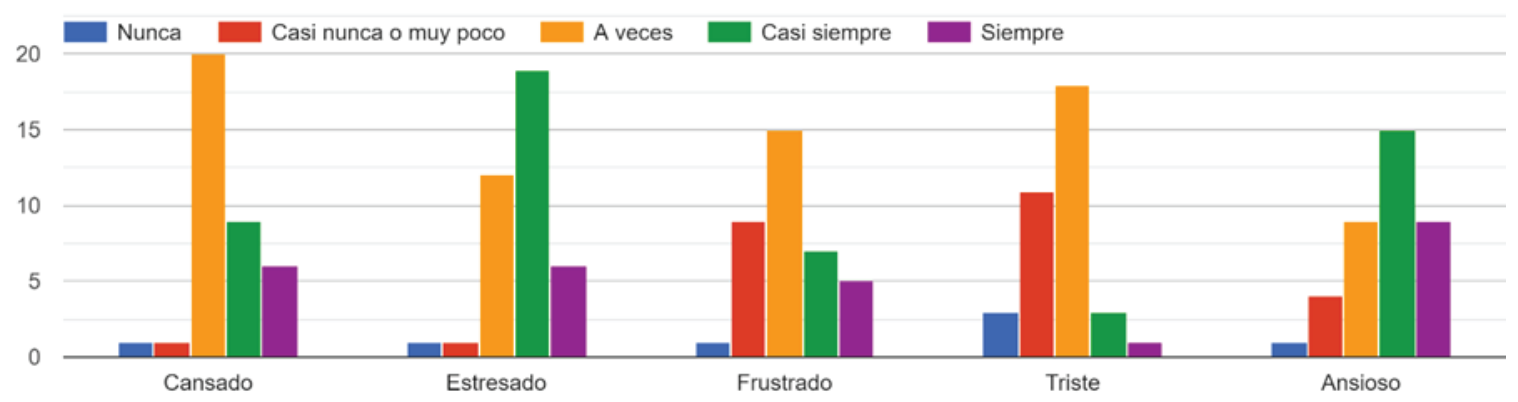

Figura 2. Percepción sobre las emociones experimentadas por los estudiantes durante las clases virtuales en pandemia

Elaboración propia

A partir de la siguiente escala, ¿cómo te has sentido después de tus clases virtuales?

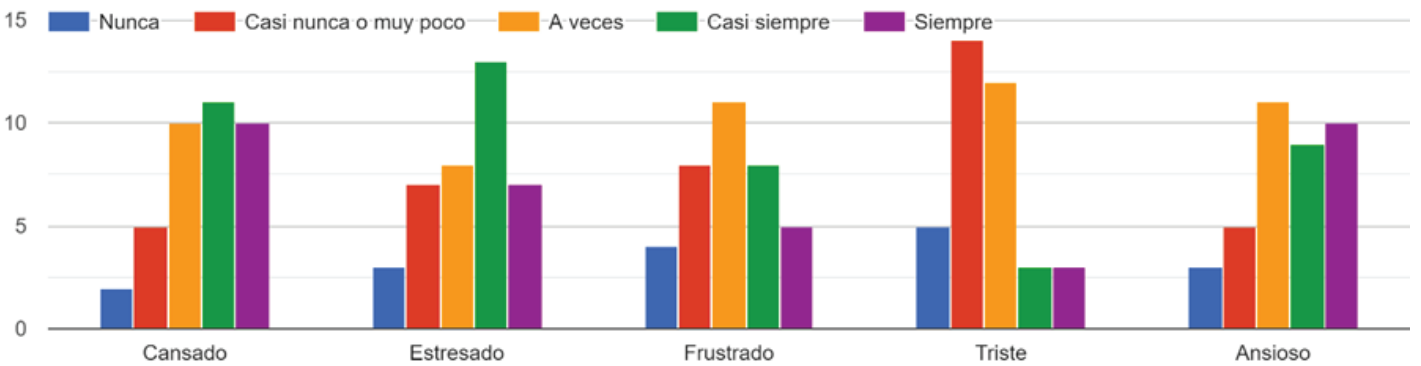

Figura 3. Percepción sobre las emociones experimentadas por los estudiantes después de las clases virtuales en pandemia

Elaboración propia 
¿Conocías sobre las pausas activas antes de que las experimentes en clase?

40 respuestas

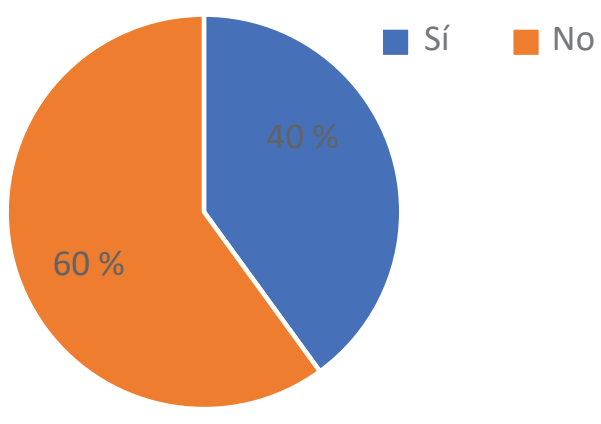

Figura 4. Cantidad de estudiantes que conocían sobre las pausas activas antes de que sean empleadas en la sesión virtual

Elaboración propia

A partir de la siguiente escala, ¿cómo te has sentido después de una pausa activa en clase clase virtual? En la escala, 1 equivale a menor grado y 5 a mayor grado.

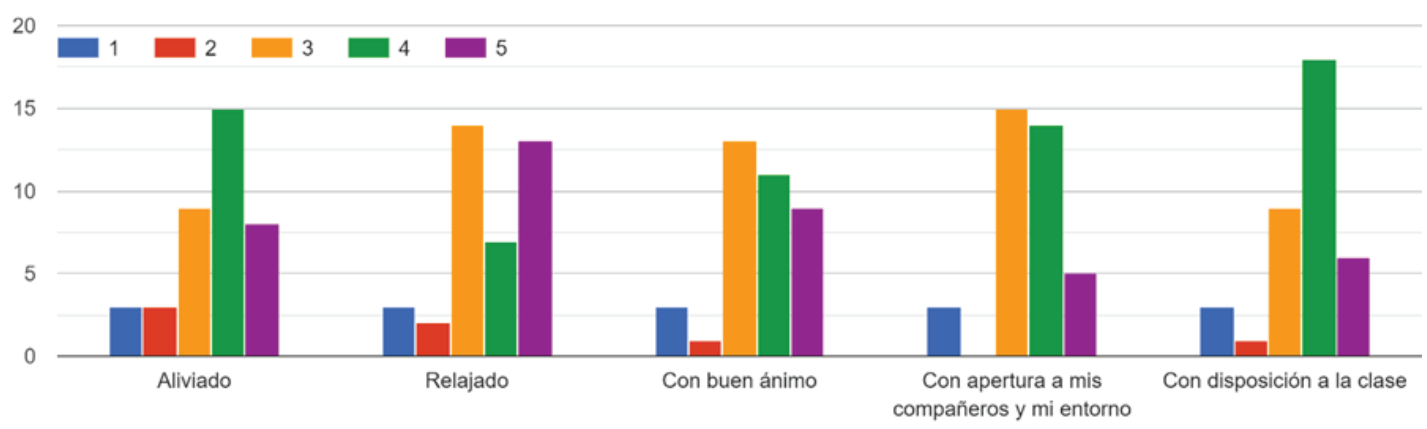

Figura 5. Escala de emociones después de una pausa activa

Elaboración propia 
¿Crees que estas pausas activas son necesarias en una enseñanza-aprendizaje virtual? 40 respuestas

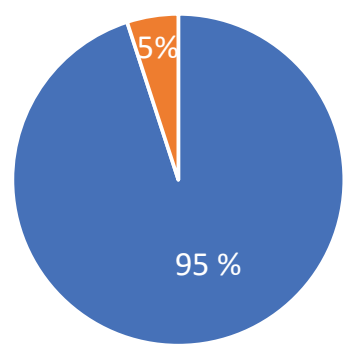

Figura 6. Cantidad de estudiantes que afirman la necesidad de las pausas activas en una clase virtual

Elaboración propia

¿Te agradaría que los profesores incorporen las pausas activas durante alguna clase virtual como estrategia pedagógica?

40 respuestas

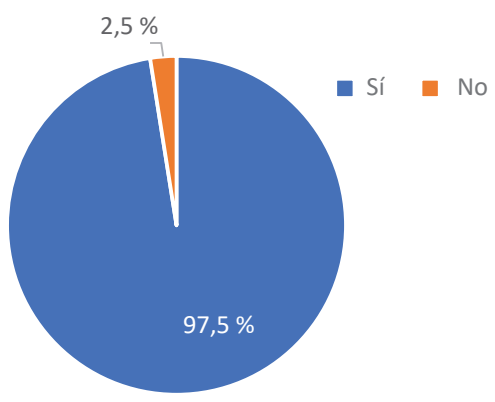

Figura 7. Cantidad de estudiantes que les agradaría que se incorporen las pausas activas en una clase virtual

Elaboración propia 
¿Crees que las pausas activas te permitieron mejorar tus competencias emocionales? 40 respuestas

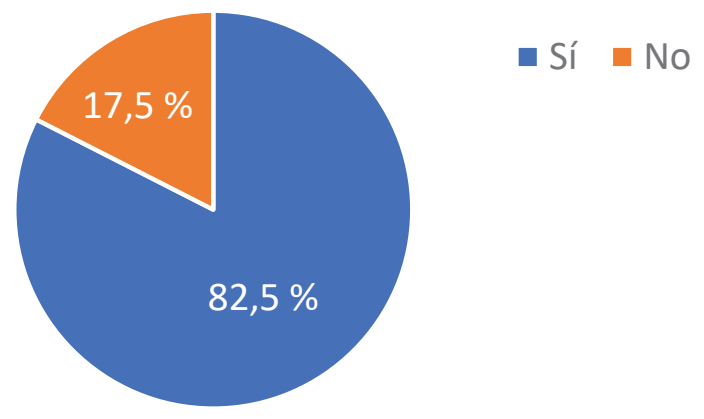

Figura 8. Cantidad de estudiantes que consideran que las pausas activas mejoraron sus competencias emocionales

Elaboración propia

¿Crees que las pausas activas te permitieron mejorar tu bienestar personal?

40 respuestas

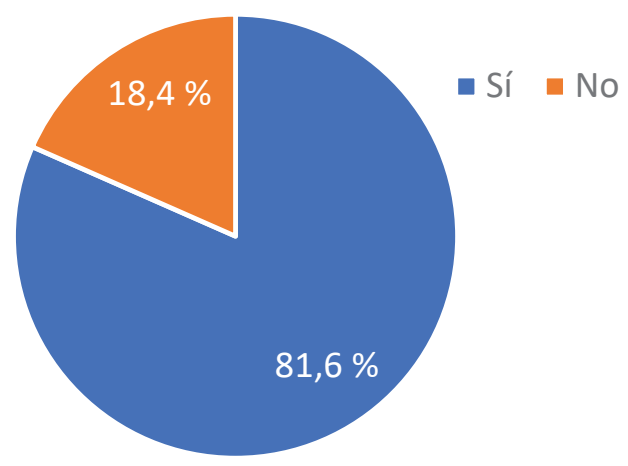

Figura 9. Cantidad de estudiantes que consideran que las pausas activas mejoraron su bienestar personal

Elaboración propia 
¿Consideras que las pausas activas mejoran el rendimiento académico?

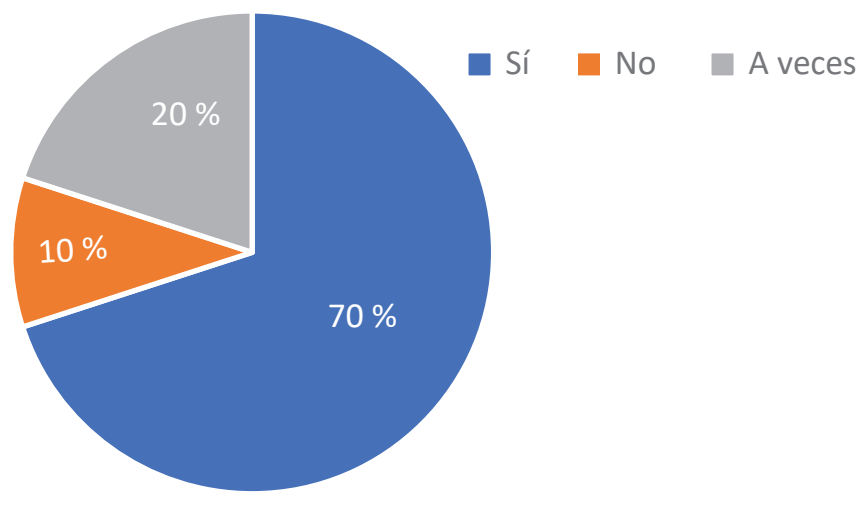

Figura 10. Cantidad de estudiantes que consideran que las pausas activas mejoran el rendimiento académico

Elaboración propia

\section{DISCUSIÓN}

La presente investigación tuvo como objetivo reconocer la importancia del uso de las pausas activas en la modalidad de enseñanza virtual para promover el bienestar emocional en jóvenes universitarios durante el periodo de pandemia.

En la encuesta, el $90 \%$ de los estudiantes revelaron haber tenido sobrecarga académica durante la crisis sanitaria (véase la figura 1). Y en este periodo, durante y después de las clases virtuales, se acentuaron emociones negativas como estrés, ansiedad, frustración, tristeza y cansancio (véanse las figuras 2 y 3 ). Es posible que estas emociones negativas se hayan desarrollado a partir de experiencias pedagógicas desarticuladas, aquellas que han sido diseñadas en un contexto regular sin contemplar las emociones de los estudiantes ante un contexto adverso. Al respecto, Arana (2020) sostiene que el ambiente de clase:

Es resultado de la relación emocional que el estudiantado tenga (o no) con la dinámica implicada y sus contenidos, y el modo en que la persona facilitadora pueda acceder a esta información y utilizarla para mejorar aspectos como la motivación, la participación o el mismo desempeño académico. (párr. 10) 
Frente a ello, es indispensable considerar el uso de estrategias pedagógicas adecuadas que permitan manejar entornos adversos. Las pausas activas como estrategias pedagógicas promueven el desarrollo de emociones positivas (véase la figura 5). Se considera emociones positivas a aquellas que posibilitan la asertividad, brindan autonomía y dominio emocional, favorecen la creatividad, potencian el crecimiento personal: la persona es consciente de su entorno y es capaz de afrontar los diversos acontecimientos. Estas emociones positivas se logran cuando las pausas activas canalizan energías, liberan la carga emocional, emplean ejercicios de motricidad y mejoran el estilo de vida. En este sentido, Arana (2020) sugiere que se deben planificar estrategias pedagógicas para propiciar un ambiente emocional, donde el participante se sienta libre y seguro de expresarse. Asimismo, agrega que esta planificación fomentará un ambiente de empatía y de cuidado con los otros en un contexto de clase virtual.

De esta manera, los estudiantes encuestados han considerado necesario aplicar las pausas activas en la enseñanza-aprendizaje virtual porque mejoran el bienestar emocional y personal (véanse las figuras 6, 7, 8 y 9). Los resultados demuestran que el uso de una adecuada gestión de las pausas activas permite al estudiantado afrontar contextos emergentes. De acuerdo con Alvarado (2021), un modelo pedagógico por competencias emocionales refuerza el dominio emocional de los estudiantes frente al contexto actual.

\section{CONCLUSIONES}

En conclusión, la enseñanza-aprendizaje en modalidad virtual actual tendría que contemplar el uso de pausas activas con la finalidad de que los estudiantes alcancen un dominio de sus propias emociones frente a un contexto adverso. Este estudio recoge las percepciones de los estudiantes de la Universidad de Lima sobre la aplicación de las pausas activas durante las clases virtuales 2020-2, un ciclo después de que ya se hubiera instaurado la modalidad virtualizada.

Se concluye que una enseñanza-aprendizaje virtual desarticulada o desatendida de las competencias emocionales de los estudiantes genera emociones negativas. Autores como Rebollo et al. (2008) ya anunciaban la relevancia de una concepción constructivista de las emociones en los recursos, contenidos y entornos educativos de aprendizaje en línea. Por esta razón, la presente investigación sugiere la implementación de pausas activas como estrategias pedagógicas, porque desarrollan habilidades emocionales o emociones positivas que permiten afrontar una experiencia pedagógica en un contexto de crisis sanitaria. 


\section{REFERENCIAS}

Alvarado, D. (2021). Educación emocional, un complemento en el proceso enseñanzaaprendizaje virtual a nivel superior durante COVID-19. Revista Scientific, 6(19), 329-348. https://www.indteca.com/ojs/index.php/Revista_Scientific/article/ view/553/1266

Arana, S. (2020). La evaluación de las emociones dentro del aula virtual universitaria en relación al aislamiento producido por el COVID-19. Centro Cultural de España en Costa Rica. http://ccecr.org/participacion/la-evaluacion-de-las-emocionesdentro-del-aula-virtual-universitaria-en-relacion-al-aislamiento-producidopor-el-covid-19/

Comisión Económica para América Latina y el Caribe; Organización de las Naciones Unidas para la Educación, la Ciencia y la Cultura. (2020). La educación en tiempos de la pandemia de COVID-19. https://repositorio.cepal.org/bitstream/ handle/11362/45904/1/S2000510_es.pdf

Florines, M. (2017). Pausas activas como herramienta didáctica de aula en educación secundaria [Tesis de licenciatura, Instituto Universitario Asociación Cristiana de Jóvenes]. http://www.accede.iuacj.edu.uy/bitstream/handle/20.500.12729/263/ TFG_2017_Florines_M.pdf?sequence=1\&isAllowed=y

Jiménez, F., y Monroy, J. (2015). Las pausas activas escolares como estrategia para influenciar la atención en los estudiantes del grado primero, de la jornada tarde del IED Francisco José de Caldas, Sede "C" [Proyecto de grado, Universidad Libre de Colombia]. https://repository.unilibre.edu.co/bitstream/handle/10901/8337/TRABAJ0\%20 FINAL\%20FREDDY\%20JIMENEZ\%20\%281\%29.pdf?sequence=1\&isAllowed=y

Lorca, P. (2016). Consideraciones pedagógicas para la planificación y ejecución de la pausa activa, una mirada desde la comuna de Valdivia, región de Los Ríos. http:// cybertesis.uach.cl/tesis/uach/2016/ffl865c/doc/ffl865c.pdf

Lovón, M., y Cisneros, A. (2020). Repercusiones de las clases virtuales en los estudiantes universitarios en el contexto de la cuarentena por COVID-19: el caso de la PUCP. Propósitos y Representaciones, 8(3), 1-15. https://revistas.usil.edu.pe/index.php/ pyr/article/view/588

Meza, B. (2010). Bienestar psicológico en practicantes de danza contemporánea [Tesis de licenciatura, Pontificia Universidad Católica de Perú]. http://tesis.pucp.edu.pe/ repositorio/handle/20.500.12404/1217

Ministerio de Educación del Ecuador. (2020). Pausa activa en las actividades escolares. Guía didáctica para docentes. https://13deabril.edu.ec/wp-content/uploads/2020/12/ pausa_activa0155088001606400649-1.pdf 
Rebollo, M. A., García, R., Barragán, R., Buzón, O., y Vega, L. (2008). Las emociones en el aprendizaje online. RELIEVE, 14(1), 1-23. https://ojs.uv.es/index.php/RELIEVE/ article/view/4201/3809

Rodríguez, P. (2016). La danza/movimiento como recurso de expresión y transformación aplicada a las competencias básicas de la educación emocional [Monografía, Universidad Distrital Francisco José de Caldas]. https://repository.udistrital. edu.co/bitstream/handle/11349/5166/RodriguezLaverdeDianaPatricia2016.pdf; jsessionid=8C0D3975F731C78442CC5EAC9C07D9F2? sequence $=1$

Siete de cada 10 peruanos han visto afectada su salud mental durante pandemia. ( 5 de julio del 2020). Andina. Agencia Peruana de Noticias Andina. https://andina.pe/ agencia/noticia-siete-cada-10-peruanos-han-visto-afectada-su-salud-mentaldurante-pandemia-video-804192.aspx 